\title{
Diagnosis of Parotid Gland Mass by the Fine Needle Aspiration Cytology (FNAC) and it's Histopathological Correlation - 2 Years Study in BSMMU, Dhaka
}

\author{
AAllam Choudhury ${ }^{1}$, Tuhin Sultana ${ }^{2}$, Belayat Hossain Siddique ${ }^{3}$, A. Sufi Ahmed Amin ${ }^{4}$ \\ ${ }^{1}$ Associate professor, Dept of Otolaryngology and Head Neck Surgery, ${ }^{2}$ Associate Professor Dept of Clinical Pathology, ${ }^{3}$ Professor, Dept \\ of Otolaryngology and Head Neck Surgery, ${ }^{4}$ Professor, Dept of Otolaryngology and Head Neck Surgery, Bangabandhu Sheikh Mujib \\ Medical University, Shahbag, Dhaka, Bangladesh
}

\begin{abstract}
:
Background: Fine needle aspiration cytology is a widely practiced technique in the diagnosis of parotid lump. Fine needle aspiration cytology (FNAC) is a simple, quick, inexpensive and minimally invasive technique used to diagnose different types of masses. In otolaryngology, FNAC's greatest utility is in the diagnosis of neck masses.Objectives: The aim of this study was to assess the sensitivity and specificity of FNAC in the diagnosis of parotid mass. Parotid gland lesions form about 2-6.5\% of all head and neck neoplasms in adults. They are easily accessible by FNAC, also cytology can provide a distinction between parotid and non-parotid lesion, benign and malignant lesions, and specific and non specific inflammation. Methods: 50 patients were studied prospectively over 2 years. FNAC was done using 10 cc syringes and 20-22 G. needle and stained with papanicular stain. Histopathology was assessed on routine H \& E (haematoxylin and eosin) stained paraffin sections. Results: Sensitivity and specificity for diagnosing malignant and benign tumours were $75 \%, 95.2 \%$, and $92.5 \%, 80 \%$, respectively, and $90 \%$ of benign tumours were accurately typed on fine-needle aspiration cytology compared with $92 \%$ in the malignant group. Conclusion: Fine-needle aspiration cytology is useful in the preoperative assessment of parotid tumours as it is more reliable than clinical examination to diagnose malignant parotid tumours. FNA cytology is useful in avoiding surgery (inflammatory lesions) or limiting surgical procedures (benign tumours).
\end{abstract}

Key words: Parotid gland tumour, Fine needle cytology

[BSMMU J 2011; 4(2):65-69]

\section{Introduction:}

Salivary gland neoplasm constitute about $10 \%$ of all head \& neck neoplasm which represent $3 \%$ of all neoplasm of the body. The value of FNAC in the investigation of salivary gland disease has been widely debated amongst clinicians and cytopathologists ${ }^{1,5}$ Fine needle aspiration cytology (FNAC) of suspected salivary gland lesions has an established role in preoperative diagnosis and management of patients. However diverse morphological patterns and overlapping features make it a challenging job, to give a precise diagnosis, at times ${ }^{2}$. The aim of the present study is to evaluate the effectiveness of FNAC in the diagnosis of parotid gland masses. Fine needle for diagnostic technique that is widely employed for lesions of the head and neck. Among head and neck sites, the parotid gland is unique in the number, diversity, and peculiarity of its pathological processes. This complexity has prompted a great deal of discussion regarding the

Address for Correspondence: A Allam Choudhury, Associate Professor, Dept of Otolaryngology and Head Neck Surgery, Bangabandhu Sheikh Mujib Medical University, Shahbag, Dhaka, Bangladesh application of FNAC to parotid masses. Primarily focusing on the reliability of FNAC as a diagnostic tool in guiding patient management. Parotid gland lesions form about 2$6.5 \%$ of all head and neck neoplasm in adults, and present as enlarged masses which are usually accessible for FNAC $^{3}$. They are not generally subjected to incisional or needle biopsy techniques because of the risks of fistula formation, or in the case of neoplasm, of tumour implantation. There is no evidence that these complications occur with FNAC ${ }^{2}$. The present study was undertaken to evaluate parotid gland lesions by FNAC and to correlate the cytologic findings with histopathology.

Cytology can clearly distinguish between parotid and non parotid lesions, benign and malignant lesions, so also specific and non specific inflammation. Thus it provides decisive direction for therapeutic management of the patient. FNAC is a utility tool for subtyping of parotid gland lesions with variable specificity and sensitivity ${ }^{4}$.

The overall accuracy of fine needle aspiration cytology was $87 \%$, false-positive and false-negative rates for malignant disease both being $4 \%$. The sensitivity, 
specificity and accuracy of fine needle cytology for malignant parotid tumours was 66\%, 95\%, and 91\%, respectively, that of benign tumours (pleomorphic adenoma or Warthin's tumour) being

88\%, 83\% and 87\%, respectively. Sensitivity, specificity and accuracy for the remaining principally inflammatory parotid diseases was $100 \%$, 95\% and 96\% respectively. The predictive value of a positive test for malignant tumours, benign tumours and inflammatory conditions was 66\%, 94\% and 75\%, respectively. The negative predictive value for these conditions was $95 \%, 71 \%$ and $100 \%$, respectively ${ }^{3}$.

The benefit of fine needle aspiration cytology in parotid diseases is debated, some claiming it alters clinical decision making in over one third of cases ${ }^{1,4}$. Improvements in radiological assessment have occurred with the use of ultrasound and CT scanning, nuclear magnetic resonance imaging and digital subtraction sialography ${ }^{6}$.

Fine-needle aspiration cytology (FNAC) has gained widespread acceptance and popularity among head and neck surgeons in the assessment of thyroid and neck masses but its use in the evaluation of parotid tumours has not attained similar enthusiasm. The main reason in the belief that the presence of a paroitid lump is an indication for its removal. ${ }^{7}$ Further more, the sensitivity and specificity of FNAC for parotid tumours is between 57-98\% and 86-100\%, respectively, and hence, some authors believe that it is not accurate enough to influence the decision-making process. ${ }^{8}$ The objective of this study is to assess the sensitivity and specificity of FNAC in the diagnosis of malignant and benign neoplasms and to evaluate its usefulness in an algorithm in the management of parotid tumours.

If the correlation of FNAC and histological diagnosis is significant then by doing the preoperative cytological diagnosis the clinician can start the initial treatment and plan the mode of treatment. Also it can prevent excess morbidity associated with over treatment

\section{Methods:}

This a prospective study done in Department of Otolaryngology-Head \& Neck Surgery and Pathology, BSMMU from July 2007 to August 2009.50 cases of parotid mass were selected by method of randomization which were later on operated. FNAC were carried out in 50 cases preoperatively and findings were correlated with final histopathological report. Detailed clinical history, results of local examination, general examinations and systematic examination were recorded in each case.

FNAC was done using 10 cc syringes with 20-22 G. needles after taking informed consent of the patient. Smears were stained with papanicolous stain. Excisional biopsy specimens were fixed in 10\% formalin. Gross and microscopic examination were performed in each case. H\& E stain was done in all cases.

Fine-needle aspiration cytology results were classified into the following categories: benign, malignant and nonneoplastic lesions.

The corresponding cytological and histological diagnosis were reviewed retrospectively and analysed. Study design included a comparison between results of preoperative FNAC with final histopathological diagnoses. Data analysis was calculated sensitivity and specificity of FNAC in differentiating between benign and malignant lesions.

\section{Results:}

50 patients having both preoperative FNAC and final histopathological diagnosis constituted the study group. There were 32 men and 18 women in an age range of 12-77 years (mean 48.7 years). 41 patients underwent superficial parotidectomy, and 9 total parotidectomy.

Most of the benign tumour are pleomorphic adenoma (68\%) Fig-1 shows a patient of pleomorphic adenoma of parotid gland. Fig-2 shows fine needle aspiration cytology picture of pleomorphic adenoma.

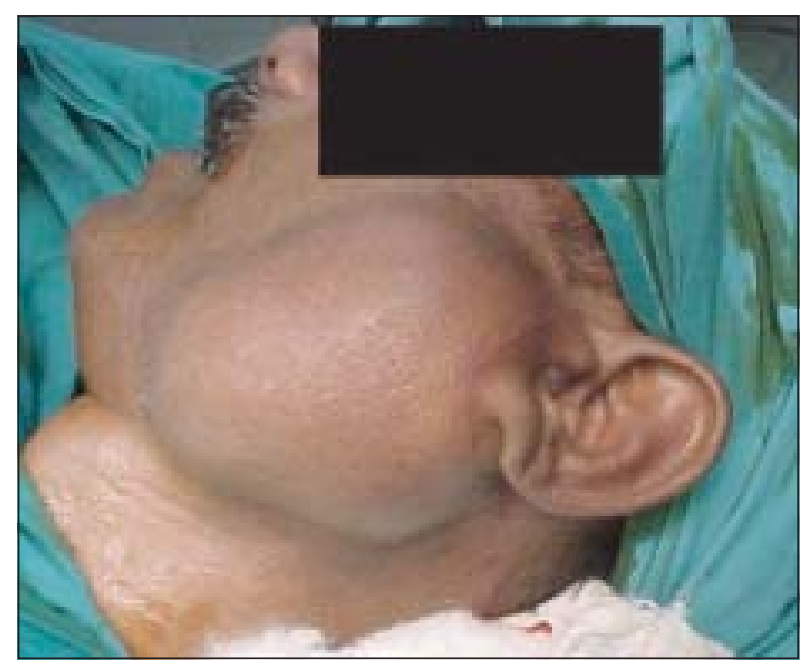

Fig-1: Pleomorphic adenoma of parotid gland. 


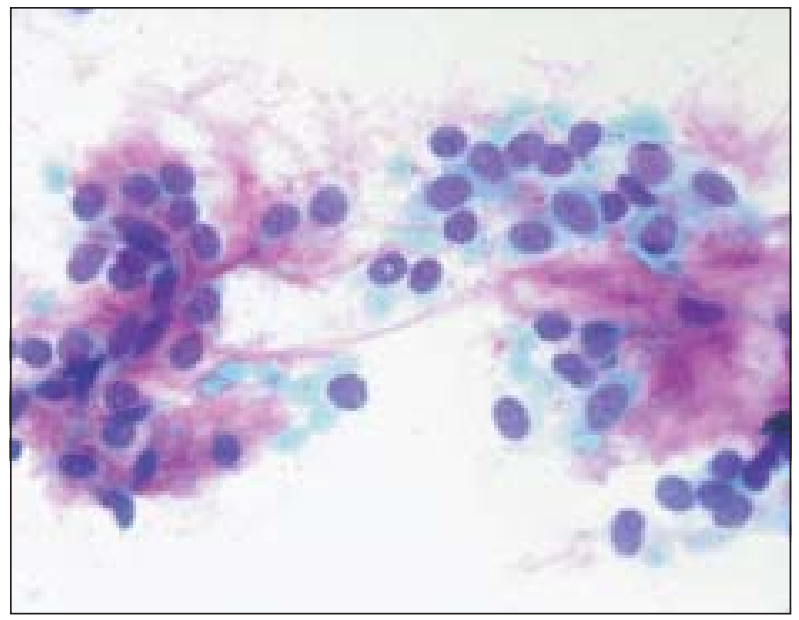

Fig-2: FNAC picture of pleomorphic adenoma.

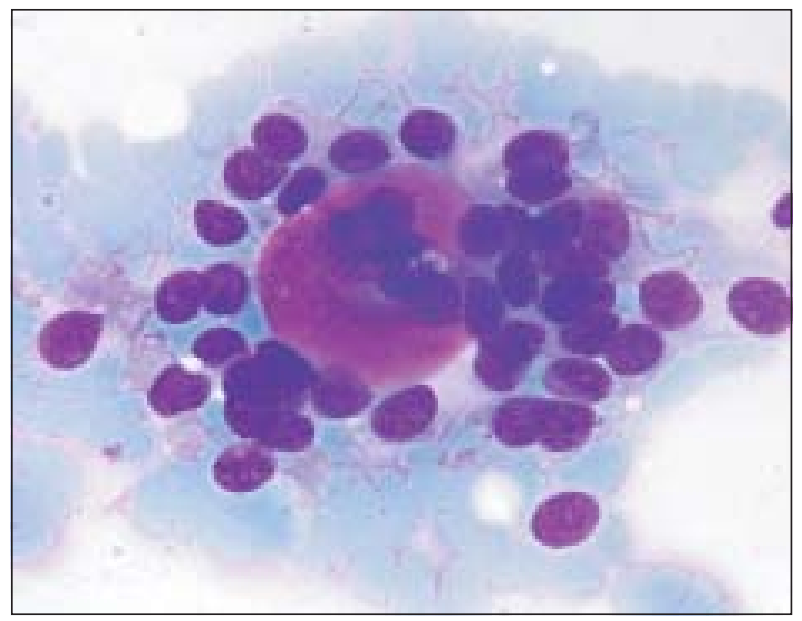

Fig-3: FNAC picture of adenocystic carcinoma

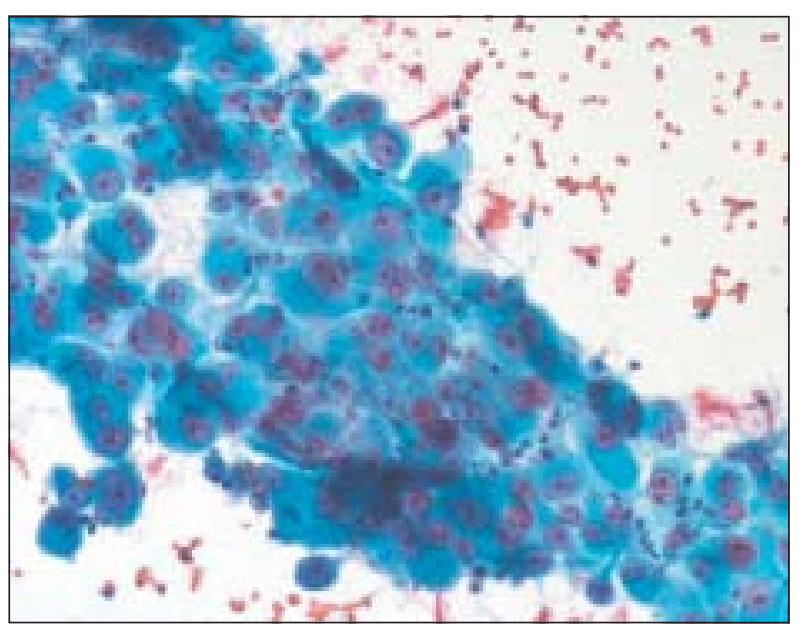

Fig-4: FNAC picture of mucoepidermid carcinoma.

Most of the malignant tumours are adenocystic (10\%) \& then mucoepidermoid (6\%) carcinoma. Fig-3 \& 4 show adenocystic and mucoepidermoid carcinoma FNAC picture respectively.

The results of FNAC showed that 39 (78\%) were benign, $9(18 \%)$ were malignant and 2(4\%) non-neoplastic lesion. Final histological diagnosis confimed the presence of 8 (16\%) malignancies, 40 (80\%) benign tumours 2 (4\%) nonneoplastic lesion. (Table-I).

Table-I

Histopathological \& cytological diagnoses of 50 cases

\begin{tabular}{lcl}
\hline Benign & $\begin{array}{c}\text { Histological } \\
\text { diagnosis }\end{array}$ & $\begin{array}{l}\text { Cytological } \\
\text { diagnosis }\end{array}$ \\
Pleomorphic Adenoma & $80 \%$ & $78 \%$ \\
Warthin's & 35 & 33 (2 adenocystic carcinoma $)$ \\
Lipoma & 4 & 3 (1 diagnosed as tubercular lesion) \\
Malignant & 1 & 1 \\
Carcinoma in pleomorphic adenoma & $16 \%$ & $18 \%$ \\
Adonocystic carcinoma & 1 & $(1$ diagnosed as pleomorphic adenoma) \\
Mucoepidermoid carcinoma & 4 & $3(1$ Diagnosed as malignant neoplasm lof uncertain type \\
Non-neoplastic & 3 & 3 \\
Benign lymphoepithelial cyst & $4 \%$ & $4 \%$ \\
Tubercular intraparotid lymph node & 1 & 1 \\
Total & 1 & $(1$ diagnosed as Warthin’s tumour $)$ \\
\hline
\end{tabular}


Fine-needle aspiration cytology did not correlate with 6 specimens of which 3 were benign, 3 were malignant. So accuracy of benign tumour by FNAC was $90 \%$ \& malignant tumour was $92 \%$.

Sensitivity and specificity of benign tumour 92.5\% and 80\% \& malignant tumour 75\% \& 95.2\%. So cytological diagnosis were $88 \%$ correlate with histological diagnosis. (Table-II).

\section{Table-II}

Histopathological \& cytological analysis of 50 cases.

\begin{tabular}{lccc}
\hline & Sensitivity & Specificity & Accuracy \\
\hline Benign & $92.5 \%$ & $80 \%$ & $90 \%$ \\
Malignant & $75 \%$ & $95.2 \%$ & $92 \%$ \\
\hline
\end{tabular}

\section{Discussion:}

Fine-needle aspiration (FNA) is a safe diagnostic technique that is widely employed in the examination of parotid masses at relatively low cost and minimal risk to the patient. Preoperative diagnosis of benign and malignant tumour are important for the surgeons. The standard surgical treatment for most benign tumours in the superficial lobe is lateral parotidectomy, while benign tumours in the deep lobe are usually treated by total parotidectomy with facial nerve preservation. ${ }^{6}$ Conservative surgical modalities for benign tumours, such as extracapsular dissection, partial lateral parotidectomy, and deep lobe parotidectomy with preservation of the superficial lobe, have been discussed in the literature. ${ }^{7,9}$ Early primary parotid carcinomas of the superficial lobe are treated in many centres by total parotidectomy, while superficial parotidectomy is performed in other centres. Elective neck dissection for primary parotid carcinomas of the clinically negative neck is performed routinely by only a few authours. ${ }^{2}$ However, most authors advocate neck dissecion on the basis of the histological type of the carcinoma and tumour grade.

Therefore, Selection of appropriate surgical procedure is dependent upon whether a tumour is benign or malignant on preoperative cytological diagnosis. In the present study, male preponderance was observed. There were 40 (80\%) benign neoplastic cases. The incidence of benign neoplasm has been reported as $40 \%, 61 \%$ and $69 \%$ by different authors. $3,4,616 \%$ of our cases were malignant lesions as against 6\%,37\% and 13\% reported by other authors $(2,4,6) .4 \%$ percent of cases were non neoplastic. Most common benign tumour was pleomorphic adenoma and most common malignant tumour was adenoid cystic carcinoma in this study. Among the parotid malignancy, 3 were mucoepidermoid carcinoma \& 1 was carcinoma in pleomorphic adenoma. Mucoepidermoid tumour in this study was the second common malignant tumour which correlates with others study. ${ }^{2.8}$

Neoplasm of salivary gland may occur at any age, Marshall and Miles (1974) showed malignant tumours usually appear in later age group but may be seen in the adolescents. In this study highest number of patients were in the $5^{\text {th }}$ decade $(26 \%)$ which correlate with other studies. 2,7 Regarding the sex distribution of different neoplasms, benign parotid tumours was more common in male (M:F=1.8:1). malignant parotid neoplasms was also more common in male $(\mathrm{M}: \mathrm{F}=1.8: 1)$.

The benign tumour generally have no pain or other distressing symptoms for which they do not care for it. Moreover they fear for the operative treatment. In under developed countries like us, due to poor socioeconomic conditions and non availability of modernized hospital facilities nearby-patient often report to local quackes and village doctors for their treatment before attending to a concerned specialist, for this reasons patient often reports late and sometimes with complication of the disease. in present series, most patients reported-within 4 to 8 years of the diseases. In the present series, all the cases presented with swelling. Size of the swelling in most pleomorphic adenoma were more than $2 \mathrm{~cm}$ and malignant tumours varied between 2 to $4 \mathrm{~cm}$. Patient with longer duration \& larger swelling presented in more advanced stage. Most of the patient of malignant tumours admitted in the hospital at stage- 3 is supported by others. ${ }^{8}$

Regarding investigation in present series, FNAC was done in all 50 cases (100\%). out of which 44 cases of cytological diagnosis correlate with histological diagnosis. Post operatively 50 cases were confirmed by histopathological examination.

In cases of Warthin's tumour, Cristallinins showed 90.1\% FNAC correlation with histopathological examination ${ }^{10}$. In our series, we diagnosed 4 cases of Warthins tumour by FNAC and one case was diagnosed as tubercular lesion. ${ }^{3}$ Later on 4 cases were confirmed as Warthin's tumour on histopathology. In Cristallinins study, a diagnosis of non neoplastic lesion was given in two cases, which tuned out to be Warthin's Tumour on histology. ${ }^{9}$ But in this study, out of two non neoplastic lesions one was diagnosed as Warthin's tumour on histopathology. 
In this study sensitivity and specificity for diagnosing malignant and benign tumours were $75 \%, 95.2 \%$, and $92.5 \%, 80 \%$, respectively, and $90 \%$ of benign tumours were accurately typed on fine-needle aspiration cytology compared with 92\% in the malignant group. Accuracy of benign tumour $90 \%$ and malignant tumour $92 \%$.

Other study shows sensitivity and specificity of malignant and benign were $80 \%, 100 \%$ \& 98.5\% 87.5\%, ${ }^{1} 66 \%, 95 \%$ \& 88\%, 83\% respectively. ${ }^{2}$ FNAC has a sensitivity \& specificity of neoplastic lesion $94.54 \%, 80.95 \%,{ }^{6}$ and $79 \%$, $84 \%$ respectively. ${ }^{3}$ In some report, the accuracy rate for detecting malignant parotid tumours ranged from $79 \%$ to $97 \%$, sensitivity from $54 \%$ to $95 \%$, and specificity from $86 \%$ to $100 \% .{ }^{9}$ Many authors have reported its usefulness as a diagnostic tool, with documented accuracy rates ranging from $97 \%$ to $98 \%$ for the large and diverse group of neoplastic and inflammatory lesions involving the parotid gland, 1,5 Recent studies have reported overall accuracy rates of FNA for parotid masses ranging from $90 \%$ to $95 \%$ well within the range of $81 \%$ to $98 \%$ established in earlier studies. In fact, Das et al, reported that FNAC recognised the malignant nature of only $60 \%$ of salivary gland carcinomas, even though the specificity and accuracy of FNAC were excellent 95\% and 90\% respectively. In some comparative study, Zbaren et al. analysed 110 parotid tumours, 68 malignancies, and 42 benign tumours and reported that the accuracy, sensitivity, and specificity of FNAC for detecting malignant tumours were $97 \%$, $74 \%$ and $88 \%$, respectively. ${ }^{10}$

Regardless of one's position regarding the use of FNAC as a routine or selective tool for evaluating parotid masses, utilisation should be moderated by a number of general principles. First, the primary value of FNAC is to establish the need for definitive surgery, not to establish a specific diagnosis. Second, for decisions regarding the extent of parotid surgery, such as whether to spare or sacrifice the facial nerve, FNAC diagnosis plays a subordinate role to intraoperative findings. Third, in those instances in which cytological and clinical impressions deverge, intraoperative frozen section findings remain an important arbitrator.

FNAC is a safe, cost effective, quick and easy diagnostic procedure that causes little discomfort to the patient. Fine needle aspiration cytology is a valuable adjunct to preoperative assessment of parotid masses as save, noninvasive procedure, almost without contraindications. The high rate of specifity of FNAC presents low possibility that benign cytological diagnosis of parotid tumors become malignant in final histopathological diagnosis. Preoperative recognition of malignant tumors may help prepare both the surgeon and patient for appropriate surgical procedure.

\section{Conclusion:}

The use of FNAC in the pre-operative evaluation of the patient with a parotid mass is likely to influence management where the clinical features suggest the possibility of a benign or malignant. Fine-needle aspiration cytology is useful in the preoperative assessment of parotid tumours as it is more reliable than clinical examination along to diagnose malignant parotid tumours. Although it may not accurately type the malignant tumours, the diagnosis of malignant tumours preoperatively may allow for appropriate surgical planning by the surgeon.

FNAC was found to be simple, noninvasive and cost effective and rapid diagnostic tool for parotid gland lesions. It plays a key role in evaluation of parotid gland tumours thus helping further surgical management of the patient.

We recommend that, where clinical teams use the results of FNAC to influence management of a primary parotid neoplasm, caution should be exercised and on going audit of the performance of FNAC is required within each institution.

\section{References:}

1. Chwee ML, Juliana T, Kwok SL, Siew SC, Lynne HYL, Luke KST. 'Role of fine-needle aspiration cytology in the evaluation of parotid tumours'. ANZJ. Surg, 2007; 77: 742-744.

2. Gordon TD, Kevin B, Roy A J S. An audit of surgery of the parotid gland. Ann R Coll Surg Engl, 1995; 77: 188-192.

3. Balakrishnan K, Mcmahon J, Imric J, Feeley KM, Parker AJ, Bull PD. Fine needle aspiration cytology in the management of a paroitid mass. A two centre retrospective study. The surgeon, 2005; 3:2: 67-72.

4. Howlett DC. Diagnosing a parotid lump: fine needle aspiration cytology or core biopsy?. The british journal of Radiology, 2006;79: 295-297.

5. Kotwal M, Gaikwad S, R Patil, Munshi M, Bobhate S. FNAC of salivary gland - a useful tool in preoperative diagnosis or a cytopathogist's riddle?. Journal of Cytology, 2007;24:2:85-88.

6. Khandekar MM, Kavatkar AN, Patankar SA, Bagwan IB, Puranik SC, Deshmukh SD. 'FNAC of salivary gland lesions with histopathological correlation. India Journal of Otolaryngology and Head and Neck Surgery, 2006;58:3:246248.

7. Mohammed SI, Azharul I, Abdus S, Ekramuddula AFM, Hossain I H. 'Malignant salivary gland neoplasm clinicopathological study. Bangladesh J of Otorhinolaryngology, 2008;14:1:1-5.

8. Attilio CS, Paolo C, Pierantonio B. Marco C. 'Usefulness of fine-needle aspiration in parotid diagnostics. Oral Maxillofac Surg, 2009;13:185-190.

9. Cristallinin EG, Ascani S, Farabi R. Fine Needle aspiration biopsy of salivary gland. Acta Cytol, 1997;41:5: 1421-1425

10. Ademar T J, Danyel E C, Jose M, Oslei P A, Luiz P K. Giant pleomorphic adenoma of the parotid gland. Med Oral Patol Oral Cir Bucal, 2008; 13:1. 58-60. 